\title{
Development and antimicrobial susceptibility studies of in vitro monomicrobial and polymicrobial biofilm models with Aspergillus fumigatus and Pseudomonas aeruginosa
}

\author{
Elias K Manavathu ${ }^{1,2}$, Dora L Vager ${ }^{1}$ and Jose A Vazquez ${ }^{1,2^{*}}$
}

\begin{abstract}
Background: Mixed microbial infections of the respiratory tracts with P. aeruginosa and A. fumigatus capable of producing biofilms are commonly found in cystic fibrosis patients. The primary objective of this study was to develop an in vitro model for $P$. aeruginosa and $A$. fumigatus polymicrobial biofilm to study the efficacy of various antimicrobial drugs alone and in combinations against biofilm-embedded cells. Simultaneous static cocultures of $P$. aeruginosa and sporelings were used for the development of in vitro $P$. aeruginosa-A. fumigatus polymicrobial biofilm in SD broth in 24-well cell culture plates at $35^{\circ} \mathrm{C}$, and the biofilm formation was monitored microscopically and spectrophotometrically. Using P. aeruginosa-A. fumigatus sporelings cocultures we examined the effects of various antimicrobial drugs alone and in combination against polymicrobial biofilm by CFU and tetrazolium reduction assays.
\end{abstract}

Results: In simultaneous static cocultures $P$. aeruginosa cells killed $A$. fumigatus conidia, whereas the bacterial cells showed no substantial fungicidal effect on sporelings grown for $12 \mathrm{~h}$ or longer at $35^{\circ} \mathrm{C}$. Monospecies cultures of $P$. aeruginosa produced loosely adhered monomicrobial biofilm and addition of $10 \%$ bovine serum to the growth medium inhibited the formation of monomicrobial biofilm by P. aeruginosa whereas it produced tightly adhered polymicrobial biofilm in the presence of A. fumigatus mycelial growth. A. fumigatus produced firmly adherent monomicrobial and polymicrobial biofilms. A comparison of CFU and MTT assays showed that the latter is unsuitable for studying the effectiveness of antimicrobial treatment against polymicrobial biofilm. Tobramycin alone and in combination with posaconazole was highly effective against monomicrobial and polymicrobial biofilms of $P$. aeruginosa whereas cefepime alone and in combination with posaconazole showed excellent activity against monomicrobial biofilm of $P$. aeruginosa but was less effective against polymicrobial biofilm. Monomicrobial and polymicrobial biofilms of $A$. fumigatus showed similar susceptibility to posaconazole with and without the antibacterial drug.

Conclusions: Simultaneous static coculture of A. fumigatus sporelings grown for $12 \mathrm{~h}$ or longer was superior to ungerminated conidia with $P$. aeruginosa for the development of $A$. fumigatus- $P$. aeruginosa biofilm. $P$. aeruginosa-A. fumigatus polymicrobial biofilm shows differential susceptibility to antimicrobial drugs whereas the susceptibility of A. fumigatus to antimicrobial drugs was unchanged.

\footnotetext{
*Correspondence: jvazquez@gru.edu

${ }^{1}$ Henry Ford Hospital, 2799 West Grand Boulevard, Detroit, Michigan 48202, USA

${ }^{2}$ Current address: Division of Infectious Diseases, Department of Medicine, Georgia Regents University, 1120 15th Street, AE 3029, Augusta, GA 30912, USA
} 


\section{Background}

Polymicrobial infection caused by multiple species of microorganisms belonging to markedly different taxonomic groups is a common occurrence in severely immunocompromised patients [1-5] as well as in individuals suffering from persistent diabetic wounds [6-9], chronic pulmonary obstructive disease [10-13], cystic fibrosis patients suffering from chronic infections [14-20] and lung transplant recipients [21-23]. The microorganisms more commonly isolated from mixed microbial infections are pathogenic bacteria and fungi. A recent retrospective study of the respiratory tract microbiology of cystic fibrosis patients revealed that their airways were colonized by multiple microorganisms, in particular Pseudomonas aeruginosa (62\% prevalence) in association with Aspergillus species [24]. The epidemiology and clinical significance of Aspergillus infection in cystic fibrosis patients have been recently reviewed [25-27]. Among the numerous Aspergillus isolates recovered from the respiratory tracts of cystic fibrosis patients, A. fumigatus is the most predominant species with a prevalence ranging from $11 \%$ to $14 \%$ in the United States [28] and as high as $60 \%$ to $78 \%$ in Europe $[29,30]$, followed by $A$. terreus. Although invasive aspergillosis can occur in persons with cystic fibrosis, particularly after lung transplantation, the most common complication of Aspergillus infection is allergic bronchopulmonary aspergillosis [31-34], a condition that causes the deterioration of lung function associated with wheezing, shortness of breath, cough and chest pain.

Given the high prevalence of $P$. aeruginosa and $A$. fumigatus colonization of the airways of cystic fibrosis patients, mixed microbial infection involving these microorganisms commonly occurs in the lungs [30,35,36] producing monomicrobial and polymicrobial biofilms. The biofilm-embedded cells are highly resistant to antimicrobial drug therapy [37-40], difficult to eradicate and often develop chronic infection that acts as a reservoir causing serious life-threatening infection in individuals with debilitated immune function. Several investigators have recently studied A. fumigatus monomicrobial biofilm using in vitro [40] and human bronchial epithelial cell culture [38] models. The aerial or surface biofilm is similar to the fungal ball often associated with aspergilloma in patients with lung cavitary lesions. The aerial biofilm made up of fungal mycelia bound together by an extracellular matrix composed of a variety of macromolecules, including galactomannan, $\alpha 1,3$-glucan, monosaccharides and polyols, melanin, proteins including major antigens and hydrophobin molecules [41]. On the other hand, Loussert et al. have recently [42] studied the composition of the mycelial extracellular matrix in vivo and found to have less complex but similar composition. The monomicrobial biofilm of A. fumigatus developed in 96- well cell culture plates and in human bronchial epithelial cell culture were resistant to antimicrobial drugs $[38,40]$. Gene expression and proteomic studies by Bruns et al. [43] showed that the 24-h biofilm expressed a greater variety of genes whereas more mature older biofilm expressed mainly specialized genes for the synthesis of extracellular matrix and secondary metabolites such as gliotoxin. Mowat et al [44] and Moree et al [45] have recently investigated the in vitro interaction of A. fumigatus with $P$. aeruginosa and demonstrated that $A$. fumigatus biofilm formation is inhibited by small diffusible molecules produced by $P$. aeruginos $a$ whereas preformed biofilm was only mildly affected. To date, very little is known about the characteristics and antimicrobial drug susceptibility of mixed microbial biofilm produced by $A$. fumigatus and $P$. aeruginosa. In this paper we describe the development and antimicrobial drug susceptibility of a simple highly reliable in vitro polymicrobial biofilm model for $A$. fumigatus and $P$. aeruginosa in 24-well cell culture plates using cocultures.

\section{Methods}

\section{Microorganisms and culture conditions}

A. fumigatus 53470 (AF53470), A. fumigatus ATCC 36607 (AF36607), P. aeruginosa 56402 (PA56402) and $P$. aeruginosa ATCC27853 (PA27853) were used in this study. AF53470 and PA56402 were clinical isolates obtained from the Microbiology Laboratory of Henry Ford Hospital in Detroit, Michigan, USA whereas AF36607 and PA27853 were commercially obtained from the American Type Culture Collection, Manassas, VA 20110, USA.

The initial AF53470 and AF36607 cultures obtained from the Microbiology Laboratory and American Type Culture Collection were subcultured on SD agar (Difco brand, Becton Dickenson Diagnostics, Sparks, MD 21152, USA) for checking the viability and purity, and subsequently stored as conidial suspension in $25 \%$ glycerol at $-80^{\circ} \mathrm{C}$. Working cultures were routinely maintained on SD agar plates at $4^{\circ} \mathrm{C}$. AF53470 and AF36607 were highly susceptible to polyenes, triazoles and echinocandins, including amphotericin B, voriconazole, posaconazole (MICs $1 \mu \mathrm{g} / \mathrm{ml}, 0.25 \mu \mathrm{g} / \mathrm{ml}$, $0.062 \mu \mathrm{g} / \mathrm{ml}$, respectively) and anidulafungin (MEC $0.031 \mu \mathrm{g} / \mathrm{ml}$ ). For preparation of conidia, cultures were grown on SD agar plates for 4 days at $35^{\circ} \mathrm{C}$ to produce large amount of conidia. The SD agar containing the mycelial growth was cut into small $\left(5 \mathrm{~mm}^{2}\right)$ pieces using a sterile spatula, transferred to a $50-\mathrm{ml}$ screw-capped conical culture tube containing $25 \mathrm{ml}$ sterile distilled water and vortexed vigorously for $2 \mathrm{~min}$ to disperse the conidia from the conidiophores. The resulting fungal suspension was filtered through 8 layers of sterile cheese cloth to remove mycelial and agar debris. The clarified conidial suspension thus obtained was standardized by hemocytometer count and 
stored at $4^{\circ} \mathrm{C}$ in the refrigerator. A. fumigatus conidia do not germinate in sterile distilled water at $4^{\circ} \mathrm{C}$ in the refrigerator and remain viable for several months, thus if required the same batch of conidial suspension can be used for several experiments.

The initial PA56402 and PA27853 cultures obtained from the Microbiology Laboratory and American Type Culture Collection were subsequently subcultured on BHI agar (Difco brand, Becton Dickenson Diagnostics, Sparks, MD 21152 , USA) for the evaluation of purity and viability. The colony purified isolates were stored in $25 \%$ glycerol at $-80^{\circ} \mathrm{C}$. Working cultures were routinely grown on $\mathrm{BHI}$ agar, stored at $4^{\circ} \mathrm{C}$ and subcultured at $37^{\circ} \mathrm{C}$ once a week to maintain viable stock cultures. PA56402 and PA27853 were highly susceptible to a variety of antibacterial drugs such as aminoglycosides, $\beta$-lactams and fluoroquinolones, including tobramycin (MIC $0.125 \mu \mathrm{g} / \mathrm{ml})$, cefepime $(\mathrm{MIC} \leq 1 \mu \mathrm{g} / \mathrm{ml}$ ) and ciprofloxacin (MIC $\leq 0.25 \mu \mathrm{g} / \mathrm{ml}$ ). Since PA56402 and PA27853 grew well in SD broth we used this medium for growing polymicrobial biofilms of A. fumigatus and $P$. aeruginosa in mixed cultures. One $\mathrm{ml}$ aliquots of the overnight cultures were centrifuged in a microcentrifuge at top speed for $2 \mathrm{~min}$ and the pellets were washed 3 times ( $1 \mathrm{ml}$ each) with sterile distilled water, resuspended in $1 \mathrm{ml}$ fresh SD broth, standardized spectrophotometrically using a standard curve and subsequently used for various experiments. The use of SD broth was particularly convenient for biofilm development since it was commonly used to grow $A$. fumigatus cultures.

\section{Biofilm development}

For the development of $A$. fumigatus and $P$. aeruginosa monomicrobial and polymicrobial biofilm models, we used Costar 24-well flat bottom cell culture plates [Cat. no. 3526, Corning Incorporated, Corning, NY 14831, USA]. Briefly, $1 \times 10^{6}$ A. fumigatus conidia prepared as described above were incubated in $1 \mathrm{ml} \mathrm{SD}$ broth at $35^{\circ} \mathrm{C}$ in 24-well cell culture plates for $18 \mathrm{~h}$, and allowed them to germinate and grow producing a tightly adherent monolayer of mycelial growth at the bottom of the well. The surface mycelial growth was removed using a sterile spatula and the spent growth medium was removed by aspiration with a 1-ml micropipet. The adherent mycelial layer was washed (3 times with sterile distilled water, $1 \mathrm{ml}$ each) using a $1-\mathrm{ml}$ micropipet and the wash fluid was completely removed by aspiration. One $\mathrm{ml} \mathrm{SD}$ broth was added to the mycelial growth $(18 \mathrm{~h})$ and then inoculated with $1 \times 10^{6} \mathrm{P}$. aeruginosa cells. The mixed culture was incubated at $35^{\circ} \mathrm{C}$ for either $24 \mathrm{~h}$ or $48 \mathrm{~h}$ for the development of a mixed microbial culture producing polymicrobial biofilm. At the end of the coculturing period, any remaining surface mycelial growth was removed as previously described and the mixed fungal-bacterial culture adhered to the bottom of the 24-well tissue culture plate was washed three times with sterile distilled water $(1 \mathrm{ml}$ each). The adherent layer of fungal and bacterial cells was scraped with a wet sterile swab, resuspended in $1 \mathrm{ml}$ of sterile distilled water, vortexed vigorously for 30 seconds with $0.1 \mathrm{~g}$ sterile glass beads to resuspend the cells and the biofilm growth was determined by CFU and tetrazolium reduction assays. For CFU assay, the cell suspensions were serially diluted 10 to $10^{8}$ fold and $0.01 \mathrm{ml}$ aliquots were spotted on SD agar plates containing either ciprofloxacin $(50 \mu \mathrm{g} / \mathrm{ml})$ or voriconazole $(16 \mu \mathrm{g} / \mathrm{ml})$ for selective fungal and bacterial growth. The numbers of CFUs of $A$. fumigatus and $P$. aeruginosa were determined after $24 \mathrm{~h}$ growth at $35^{\circ} \mathrm{C}$. For the development of monomicrobial biofilms, A. fumigatus conidia and $P$. aeruginosa cells were grown as monomicrobial cultures under identical conditions and assayed for fungal and bacterial CFUs.

\section{Photomicrography}

For photomicrography the monomicrobial and polymicrobial biofilms of $A$. fumigatus and $P$. aeruginosa were grown either on $22 \mathrm{~mm}$ sterile plastic microscopic cover slips (Cat. no. 12547, Fisher Scientific Company, Pittsburgh, PA) or in Costar 6-well flat bottom cell culture plates [Cat. no. 3736, Corning Incorporated, Corning, $\mathrm{NY} 14831, \mathrm{USA}]$ in SD broth at $35^{\circ} \mathrm{C}$. Briefly, the sterile plastic cover slips were placed in a Costar 6-well cell culture plate. Three $\mathrm{ml}$ aliquots of the A. fumigatus conidial suspension containing $1 \times 10^{6}$ conidia/ml were placed in each well completely covering the plastic cover slip and the cell culture plate was incubated statically at $35^{\circ} \mathrm{C}$ for $18 \mathrm{~h}$ for $A$. fumigatus conidia to germinate and form a monolayer of mycelial growth on the plastic cover slips. The spent growth medium from each well was removed and the cover slips containing the mycelial growth were washed (3 times with sterile distilled water, $2 \mathrm{ml}$ each) and inoculated with $3 \mathrm{ml}$ of SD broth containing $1 \times 10^{6} \mathrm{P}$. aeruginosa cells $/ \mathrm{ml}$. The mixed microbial culture was incubated for $24 \mathrm{~h}$ at $35^{\circ} \mathrm{C}$ for the development of $A$. fumigatus- $P$. aeruginosa polymicrobial biofilm. The plastic cover slips containing the mixed microbial growth were washed (3 times with sterile distilled water, $2 \mathrm{ml}$ each) and transferred to a clean Costar 6-well cell culture plate and stained with crystal violet $(0.04 \%)$ for $30 \mathrm{~min}$ at $35^{\circ} \mathrm{C}$. The stained cover slips were washed (4 times with sterile distilled water, $2 \mathrm{ml}$ each) and the excess water was drained. The cover slips were briefly air-dried, mounted on a standard microscopic slide using nail polish and the biofilms were photographed using a Nikon Microscope Camera System equipped with SPOT image processing computer software [46]. With the SPOT program, each Objective (10x to $100 \times$ ) of the microscope was previously calibrated using a stage micrometer as described in the SPOT 
Software User Guide (Chapter 4, pages 76 and 77). The photomicrographs shown in Figure 1 were captured using the 60X Objective providing a total magnification of 600X. To develop monomicrobial biofilms of A. fumigatus and $P$. aeruginosa, monomicrobial cultures of these organisms were grown on plastic cover slips and processed identically. To study the kinetics of $A$. fumigatus monomicrobial biofilm development from conidia, monomicrobial cultures of A. fumigatus were grown in SD broth from a conidial suspension for $0 \mathrm{~h}$ to $24 \mathrm{~h}$ in Costar 6-well cell culture plates, washed, stained and photographed as described above.

\section{Determination of the effects of antibiotics on biofilms}

Monomicrobial and polymicrobial biofilms of A. fumigatus and $P$. aeruginosa were developed in Costar 24-well cell culture plates as previously described. The biofilms were washed with distilled water (3 times, $1 \mathrm{ml}$ each) and incubated with the appropriate concentrations of antimicrobial drug(s) for $24 \mathrm{~h}$ at $35^{\circ} \mathrm{C}$. The drug-treated biofilms were washed and the adherent cultures containing either fungal or bacterial or a mixed population of fungal and bacterial cells were harvested by scraping the bottom of the wells of the cell culture plates using sterile wet swabs into $1 \mathrm{ml}$ aliquots of sterile distilled water.

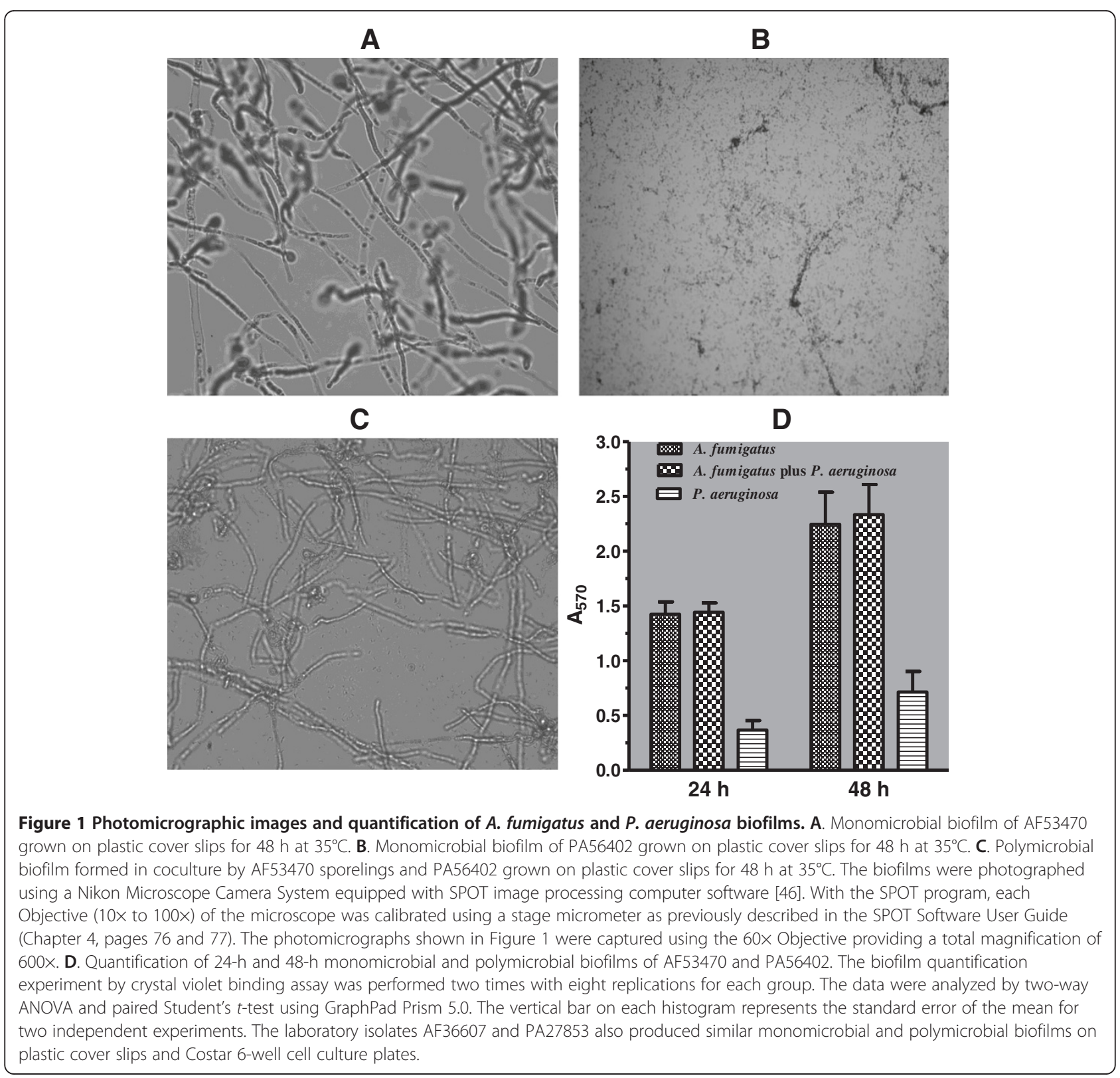


The cell suspension was vortexed vigorously with sterile glass beads to disperse the cells, serially diluted 10 to $10^{8}$ fold and $0.01 \mathrm{ml}$ aliquots of the cell suspensions were plated on ciprofloxacin $(50 \mu \mathrm{g} / \mathrm{ml})$ or voriconazole $(16 \mu \mathrm{g} / \mathrm{ml})$ containing SD agar plates and incubated for $24 \mathrm{~h}$ at $35^{\circ} \mathrm{C}$ for selective growth. The number of CFUs for each group was determined and plotted against the drug concentration to assess the effectiveness of antibiotic treatment against biofilm bound cells.

One of the disadvantages of using CFU assay to determine the growth of filamentous fungi is the poor correlation between biomass and CFU values. We therefore performed a pilot experiment where $1 \times 10^{6}$ conidia were germinated in 24-well cell culture plates in $1 \mathrm{ml}$ $\mathrm{SD}$ broth at $35^{\circ} \mathrm{C}$ form $0 \mathrm{~h}$ to 24 and the fungal growth was determined by CFU assay. The number of CFUs obtained was more or less correlated with the number of conidia, germinated conidia and sporelings grown for up to $12 \mathrm{~h}$. But once the hyphae grew extensively producing a mycelial biomass the correlation is usually reached a plateau and remained unchanged because of the geometry of the fully grown mycelial biomass and the pluripotent nature of the vegetative hyphae. Thus, the CFU assay for mature hyphae is at best an under estimation of the total fungal biomass. Since our experiments were designed to compare untreated drug-free controls to drug-treated experimental groups, determination of the absolute fungal biomass was not essential for demonstrating comparative effect of the drug treatment.

\section{Tetrazolium reduction assay}

In addition to CFU assay, we evaluated the effects of antimicrobial drugs on monomicrobial and polymicrobial biofilms of $A$. fumigatus and $P$. aeruginosa by the tetrazolium reduction assay $[47,48]$. Briefly, monomicrobial and polymicrobial biofilms of $A$. fumigatus and $P$. aeruginosa were washed three times with sterile distilled water $(1 \mathrm{ml}$ each) and the excess water was removed by aspiration with a $1 \mathrm{ml}$ micropipet. The washed adherent biofilm was overlaid with $1 \mathrm{ml}$ fresh SD broth containing $100 \mathrm{mM}$ 3-(4,5-dimethyl-2-thiazolyl)-2,5-diphenyl-2Htetrazolium bromide [MTT] and $0.2 \mathrm{mM}$ menadione and incubated at $35^{\circ} \mathrm{C}$ for $3 \mathrm{~h}$ for the reduction of the tetrazolium compound. Under these conditions, the lightly yellowish MTT will be reduced to an insoluble blue tetrazolium salt accumulated within the mycelia. At the end of the incubation period, the growth medium containing MTT was removed and the biofilm was washed three times (1 $\mathrm{ml}$ each) with sterile distilled water, and intracellular insoluble tetrazolium salt was dissolved in $1 \mathrm{ml} 70 \%$ ethanol containing $0.1 \mathrm{~N} \mathrm{HCl}$ for $30 \mathrm{~min}$ at $35^{\circ} \mathrm{C}$. The amount of intracellular tetrazolium salts was quantified spectrophotometrically by measuring the absorbance of the solution at $570 \mathrm{~nm}$. The accumulation of tetrazolium salt by the reduction of MTT by cellular dehydrogenases is proportional to the number of viable cells present in the biofilm. The effectiveness of the antimicrobial drug treatment was assessed on the basis of diminished tetrazolium reduction.

\section{Antimicrobial drugs}

Pharmaceutical grade cefepime (Sagent Pharmaceuticals, Schaumberg, IL, USA) and tobramycin pure powder were obtained from the Henry Ford Hospital Pharmacy and Sigma Chemical Company, St. Louis, USA, respectively. Stock solutions $(1 \mathrm{mg} / \mathrm{ml})$ of the antibiotics were prepared in sterile distilled water and stored as $0.25 \mathrm{ml}$ aliquots at $-20^{\circ} \mathrm{C}$. Voriconazole and posaconazole were obtained from Pfizer Pharmaceuticals (New York, NY, USA) and Schering-Plough Research Institute, Kenilsworth, NJ, USA (now part of Merck), respectively. The triazoles were dissolved in dimethylsulfoxide to obtain a stock solution of $10 \mathrm{mg} / \mathrm{ml}$ and stored as $0.25-\mathrm{ml}$ aliquots at $-20^{\circ} \mathrm{C}$. The frozen stocks of the antimicrobial drugs were thawed at room temperature and used within $24 \mathrm{~h}$. Where it is applicable, comparable concentrations of dimethylsulfoxide were used as control to examine its effect on the growth of the organism.

\section{Statistical analysis}

The data were analyzed by Student's $t$ test, one-way and two-way analysis of variance with Bonferroni's Multiple Comparison Test using Graphpad Prism Version 5.0 for Windows (GraphPad Software, Inc., La Jolla, CA, USA). A $p$ value $\leq 0.05$ was considered significant. Details of each statistical test used are given in the corresponding figure legend.

\section{Results}

\section{Germinated conidia are more suitable for polymicrobial} biofilm formation

The initial attempt for developing an in vitro A. fumigatus- $P$. aeruginosa polymicrobial biofilm model by simultaneous static coculturing of $A$. fumigatus conidia and $P$. aeruginosa cells at a cell ratio of 1:1 resulted in the complete killing of $A$. fumigatus cells. We therefore investigated the fungicidal effects of $P$. aeruginosa cell densities ranging from $1 \times 10^{1}$ to $1 \times 10^{6}$ cells $/ \mathrm{ml}$ on the survival of $1 \times 10^{6} \mathrm{~A}$. fumigatus conidia per ml after 24$\mathrm{h}$ simultaneous static coculturing. As shown in Figure $2 \mathrm{~A}$, the fungicidal activity of $P$. aeruginosa against A. fumigatus conidia was directly proportional to $P$. aeruginosa: A. fumigatus cell ratio. Ten and hundred $P$. aeruginosa cells in $1 \mathrm{ml}$ of SD broth containing $1 \times$ $10^{6}$ conidia showed very little killing of $A$. fumigatus conidia $\left(\mathrm{P}=0.5456\right.$ and 0.0871 , respectively), $1 \times 10^{3}$ and $1 \times 10^{4} \mathrm{P}$. aeruginosa cells showed moderate killing $(\mathrm{P}=$ 0.0002 and 0.0005 , respectively) whereas $1 \times 10^{5}$ and $1 \times$ 

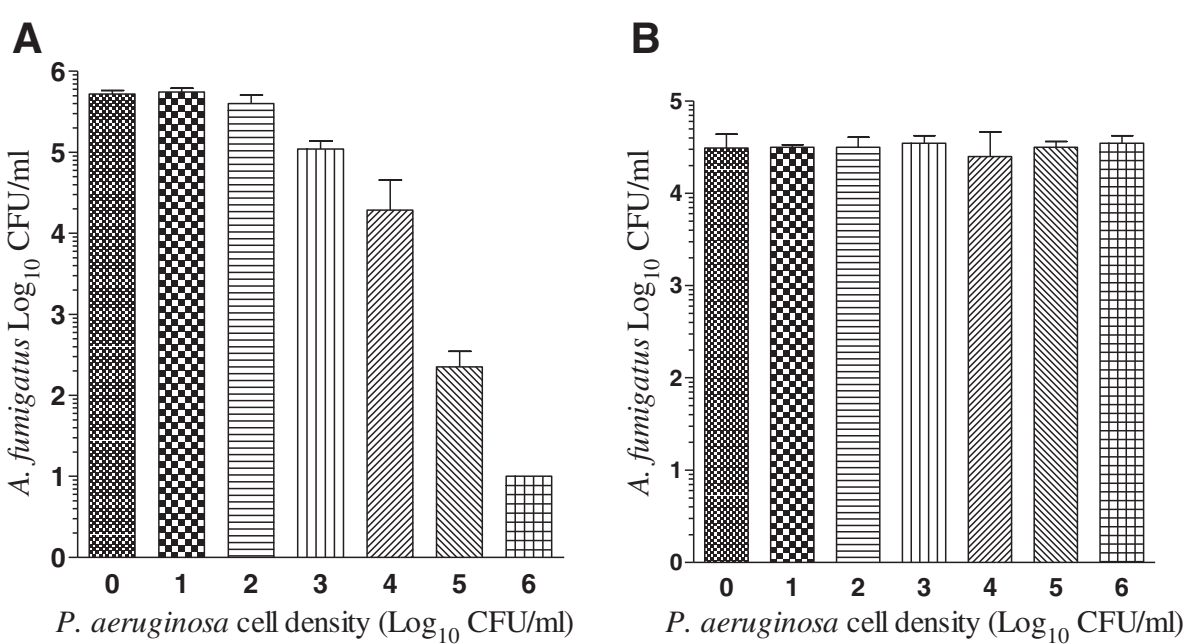

Figure 2 Effects of $\boldsymbol{P}$. aeruginosa on $\boldsymbol{A}$. fumigatus conidia (A) and sporelings (B) in cocultures. A. fumigatus conidia (A) and sporelings (B) at a density of $1 \times 10^{6}$ cells $/ \mathrm{ml}$ were incubated with $P$. aeruginosa cells ranging from $1 \times 10^{1}-1 \times 10^{6}$ cells $/ \mathrm{ml}$ in $1 \mathrm{ml} \mathrm{SD}$ broth at $35^{\circ} \mathrm{C}$ for $24 \mathrm{~h}$. At the end of the incubation the adherent microbial growth containing fungal and bacterial cells were washed 3 times with distilled water (1 ml each) and the viability of the cells was determined by CFU assay. In all mixed cultures the $P$. aeruginosa CFUs were similar $\left(\approx 1 \times 10^{10}\right.$ CFU/ml). The experiment was performed at two different times with AF53470 and PA56402 using independently prepared conidial suspensions and bacterial cultures, and one time with AF36607 and PA27853. Similar results were obtained for the clinical and the laboratory isolates. The vertical bar on each data point represents the standard error of the mean for two independent experiments with AF53470 and PA56402. The data were analyzed by one way ANOVA with Dunnett multiple comparison test where the control was compared with each of the experimental group using GraphPad Prism 5.0.

$10^{6}$ P. aeruginosa cells killed A. fumigatus conidia $99.9 \%$ and $99.99 \%$ ( $\mathrm{P}=0.0003)$, respectively. In contrast, $P$. aeruginosa cell densities ranging from $1 \times 10^{1}-1 \times 10^{6}$ cells/ $\mathrm{ml}$ did not affect the viability of $A$. fumigatus sporelings grown from a conidial suspension for $12 \mathrm{~h}$ or longer and provided more or less the same number of $\mathrm{CFU} / \mathrm{ml}$ [Figure 2B] after $24 \mathrm{~h}$ co-culturing. The lack of fungicidal activity was not because of $A$. fumigatus inhibition of $P$. aeruginosa growth since inoculation of sporelings with $1 \times$ $10^{1}$ to $1 \times 10^{6} \mathrm{P}$. aeruginosa cells $/ \mathrm{ml}$ provided approximately $1 \times 10^{10} \mathrm{P}$. aeruginosa $\mathrm{CFU} / \mathrm{ml}$ indicating that growth of $P$. aeruginosa was not affected by the presence of $1 \times 10^{6}$ A. fumigatus sporelings $/ \mathrm{ml}$. The $P$. aeruginosa cells with faster growth rate reached stationary phase in $24 \mathrm{~h}$ in the presence of $A$. fumigatus sporelings and formed a polymicrobial biofilm suggesting that a range of $P$. aeruginosa cell densities could be used to develop a polymicrobial biofilm with $A$. fumigatus sporelings.

\section{Optimum conidial density for polymicrobial biofilm formation}

It was previously shown that A. fumigatus monomicrobial biofilm formation is a function of the conidial density and production of optimum amount of biofilm was dependent on the conidial density used [40]. We therefore examined the effect of conidial density on the development of $A$. fumigatus- $P$. aeruginosa polymicrobial biofilm. As shown in Figure 3A, a plot of $A$. fumigatus conidial density ranging from $1 \times 10^{2}$ to $1 \times 10^{7}$ conidia/ml used for the mycelial growth against the biofilm associated CFUs obtained for $A$. fumigatus and $P$. aeruginosa showed that a seeding density of $1 \times 10^{6}$ conidia/ml provided the best yield of mixed microbial biofilm producing the most number of CFUs for both organisms. Although $1 \times 10^{7}$ conidia/ml produced the highest number of CFUs for A. fumigatus, the number of $P$. aeruginosa CFUs obtained was lower than that obtained when $1 \times 10^{6}$ conidia/ml was used. Among three different conidial densities $\left(1 \times 10^{4}, 1 \times 10^{5}\right.$ and $1 \times 10^{6}$ cells $/ \mathrm{ml}$ ) Mowat et al. used, $1 \times 10^{5}$ conidia/ml produced the best $A$. fumigatus biofilm in a 96-well microtiter plate [36]. The difference may be due to the difference in the surface area of the wells of 96-well and 24-well cell culture plates, or the growth media (RPMI1640 vs. SD broth) used or the assays (tetrazolium reduction vs. CFU determination) used to measure the biofilm growth.

\section{Effects of various growth media with and without bovine serum on biofilm development}

One of the primary objectives of this experiment was to identify a simple growth medium in which both $A$. fumigatus and $P$. aeruginosa would grow well and methodology for the formation of monomicrobial and polymicrobial biofilms will be simple for antimicrobial drug susceptibility testing of biofilms. The need to identify a suitable growth medium for $P$. aeruginosa biofilm formation was important because in general it produced 


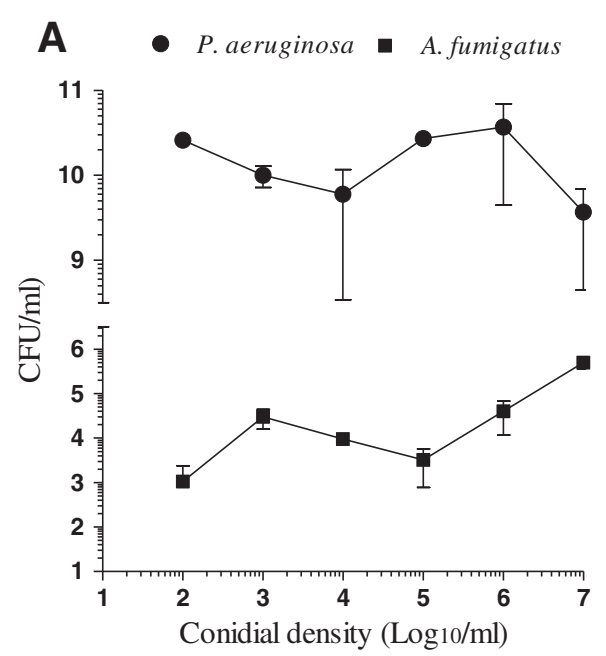

B

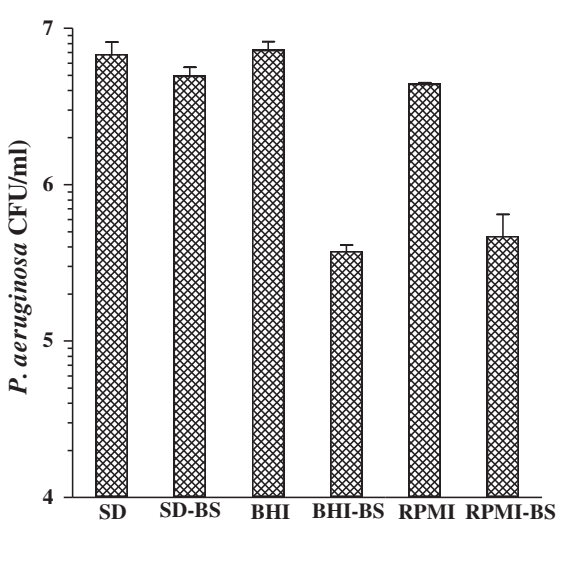

Figure 3 Effects of cell density and growth medium on biofilm formation. A. Effect of conidial density on A. fumigatus-P. aeruginosa polymicrobial biofilm formation. One $\mathrm{ml}$ aliquots of AF53470 conidial suspension containing $1 \times 10^{2}-1 \times 10^{7}$ conidia/ml were incubated in 24well cell culture plates in duplicates at $35^{\circ} \mathrm{C}$ in SD broth for $18 \mathrm{~h}$, washed and then inoculated with $1 \times 10^{6}$ PA56402 cells in $1 \mathrm{ml}$ SD broth and further incubated for $24 \mathrm{~h}$ for the development of A. fumigatus-P. aeruginosa polymicrobial biofilm. The biofilm was washed and the embedded cells were resuspended in $1 \mathrm{ml}$ sterile water and assayed for A. fumigatus and $P$. aeruginosa by CFU counts. The experiment was performed at two different times using independently prepared conidial suspensions and bacterial cultures and the vertical bar on each data point on the graph represents the standard error of the mean. B. P. aeruginosa monomicrobial biofilm formation in various growth media with and without bovine serum. One $\mathrm{ml}$ aliquots of growth media containing $1 \times 10^{6} \mathrm{P}$. aeruginosa cells were incubated in quadruplicates in 24-well cell culture plates with and without $10 \%$ bovine serum for $24 \mathrm{~h}$ at $35^{\circ} \mathrm{C}$ for biofilm formation. The adherent monomicrobial biofilm was washed (3 times), resuspended in $1 \mathrm{ml}$ sterile distilled water and the biofilm growth was assessed by CFU assay. The experiment was performed two different times with PA56402 using independently prepared bacterial cultures, and one time with PA27853. Both sets of isolates provided similar results. The data were analyzed by paired Student's $t$ test using GraphPad prism 5.0. The vertical bar on each histogram denotes standard error of the mean for two independent experiments using PA56402. Legends: SD, Sabouraud's dextrose broth; SD-BS, Sabouraud's dextrose broth with 10\% bovine serum; BHI, Brain Heart Infusion broth; BHI-BS Brain Heart Infusion broth with 10\% bovine serum; RPMI, RPMI640; RPMI-BS, RPMI1640 with 10\% bovine serum.

poor monomicrobial biofilm on plastic surfaces such as polystyrene culture plates. Since pretreatment of certain plastics with bovine serum preconditions their surfaces for better cell attachment and biofilm production $[49,50]$, we examined the effect of $10 \%$ bovine serum in the growth medium on the formation of $P$. aeruginosa biofilm. All three media we used were able to support the formation of $P$. aeruginosa biofilm to varying degree where BHI being the best medium followed by SD broth and RPMI1640 (Figure 3B). A comparison of the CFUs obtained for various media with and without bovine serum showed that the presence of $10 \%$ bovine serum inhibited $P$. aeruginosa monomicrobial biofilm formation by $27 \%$ in $\mathrm{SD}(\mathrm{P}=0.0509), 95 \%$ in $\mathrm{BHI}(\mathrm{P}=0.00016)$ and $89 \%$ in RPMI1640 ( $\mathrm{P}=0.00078)$ suggesting that bovine serum has a negative effect on $P$. aeruginosa biofilm formation in Costar cell culture plates. Thus, in our subsequent experiments, we used SD broth for the development of monomicrobial and polymicrobial biofilms of $A$. fumigatus and $P$. aeruginosa. The fact that $A$. fumigatus produces excellent monomicrobial biofilm in SD broth made it a highly suitable medium for the production of polymicrobial biofilms.

\section{Biofilm images and quantification}

Figure 1 shows photomicrographic images of 24-h monomicrobial biofilms of A. fumigatus (A), P. aeruginosa (B) and $A$. fumigatus- $P$. aeruginosa polymicrobial biofilm (C) grown on plastic cover slips. A. fumigatus produced an extensive firmly adherent mycelial growth on the plastic cover slips and in any one microscopic field only a few hyphal filaments were in focus suggesting that as the hyphae grew they branched extensively forming a network of mycelial growth producing a three dimensional structure. The monomicrobial culture of $P$. aeruginosa growing on plastic cover slips formed a loosely adhered biofilm and gentle washing did not affect its stability on the plastic cover slips. On the other hand, washing of the biofilm with agitation randomly dislodged the cells from the plastic cover slips. The mixed microbial biofilm of $A$. fumigatus and $P$. aeruginosa showed a hazy background in which numerous $P$. aeruginosa cells were embedded in a mesh-like material. In the same planar field where the bacterial cells were in clear view the fungal hyphae were out of focus and numerous bacterial cells were seen adhered to the fungal hyphae using as scaffolding forming a mixed community of microbial growth. 
Since the biofilm formation is known to increase with the duration of culturing, we investigated the effect of incubation time on the production of monomicrobial and polymicrobial biofilms of A. fumigatus and P. aeruginosa. A comparison of the amounts of crystal violet bound by 24-h and 48-h monomicrobial and polymicrobial biofilms of $A$. fumigatus and $P$. aeruginosa showed that the $48 \mathrm{~h}$ biofilm mass was increased by $57.7 \%$, $61.7 \%$ and $94.5 \%(\mathrm{P} \leq 0.0044)$ for $A$. fumigatus, $A$. fumigatus- $P$. aeruginosa and $P$. aeruginosa biofilms, respectively (Figure 1D). However, no significant difference in CFUs was obtained for 24-h and 48-h biofilms (data not shown) suggesting that CFU determination is less than suitable for the determination fungal growth in more mature biofilms (e.g., $48 \mathrm{~h}$ biofilm). However, the $24 \mathrm{~h}$ and $48 \mathrm{~h}$ polymicrobial biofilms of $A$. fumigatus- $P$. aeruginosa were almost equally susceptible to antimicrobial drugs.

\section{Drug susceptibility studies}

To examine the suitability of our in vitro biofilm model for functional studies, we investigated the effectiveness of several antimicrobial drugs individually and in twodrug combinations against monomicrobial and polymicrobial biofilms of $P$. aeruginosa and $A$. fumigatus using $\mathrm{CFU}$ and tetrazolium reduction assays. Figure 4A shows representative results for voriconazole alone and in combination with cefepime on $A$. fumigatus monomicrobial and $A$. fumigatus- $P$. aeruginosa polymicrobial biofilms as determined by the CFU assay. Voriconazole at a concentration of $32 \mu \mathrm{g} / \mathrm{ml}$ reduced the CFU of monomicrobial and polymicrobial biofilms by approximately $1.5 \operatorname{logs}$ suggesting that $A$. fumigatus cells embedded in monomicrobial and polymicrobial extracellular matrix were similarly susceptible $(\mathrm{P}=0.3681)$ to the triazole voriconazole. On the other hand, voriconazole in combination with cefepime had slightly reduced antimicrobial activity
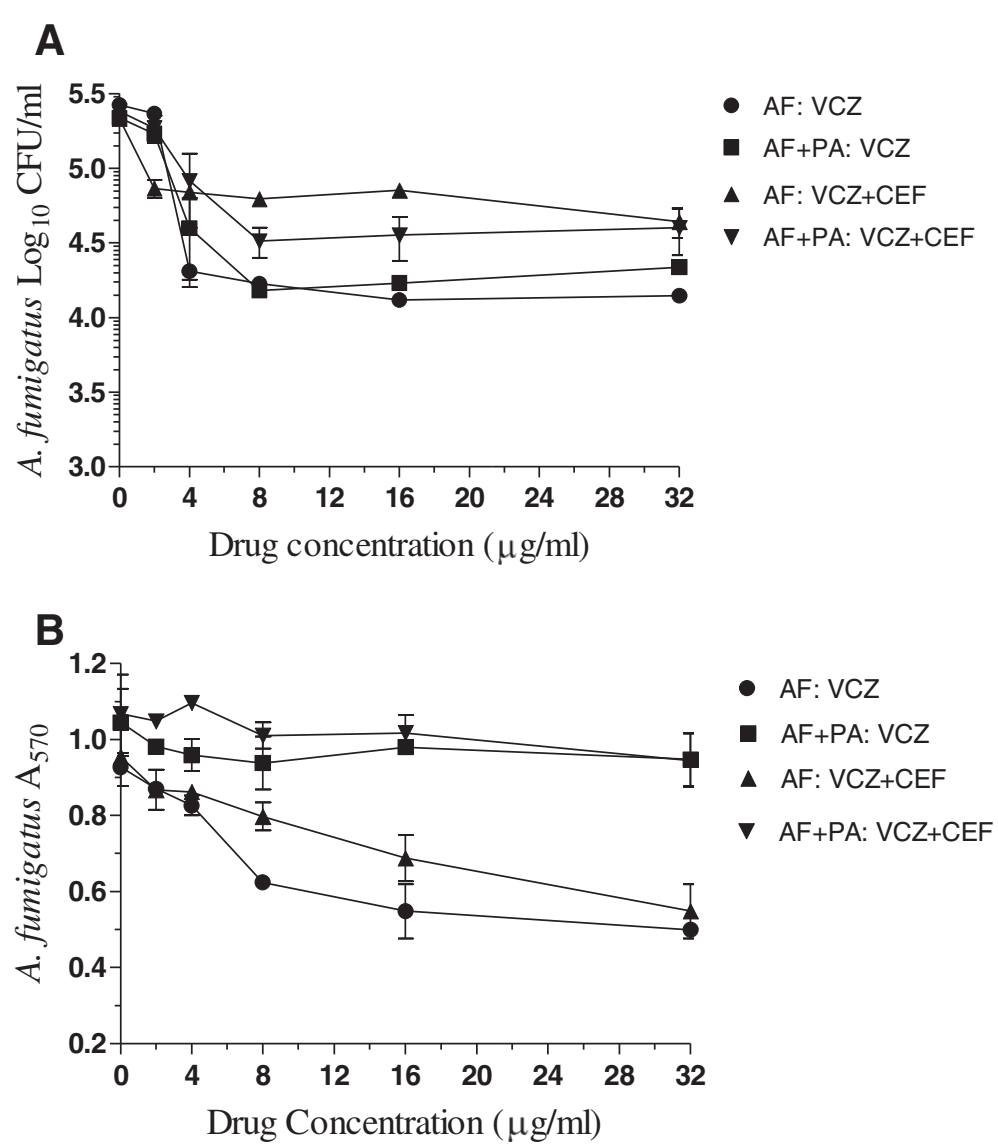

Figure 4 Effects of voriconazole alone and in combination with cefepime against $A$. fumigatus monomicrobial and $A$. fumigatus-P. aeruginosa polymicrobial biofilms as determined by CFU (A) and MTT (B) assays. The biofilms were developed in 24-well cell culture plates and the effectiveness of antimicrobial drug(s) treatment was assessed by the reduction of CFUs and $A_{570}$ values. Each experiment was performed two different times with the clinical isolates AF53470 and PA56402 using independently prepared conidial suspensions and bacterial cultures, and one time with the laboratory isolates AF36607 and PA27853. Similar results were obtained for both set of isolates. The data were analyzed by two-way ANOVA with Bonferroni post test analysis by comparing each treatment group to the other for statistical significance using Graphpad Prism 5.0. The vertical bar on each data point denotes standard error of the mean for two experiments performed with AF53470 and PA56402. Legends: AF, A. fumigatus monomicrobial biofilm; AF + PA, A. fumigatus-P. aeruginosa polymicrobial biofilm; VCZ, voriconazole; CEF, cefepime. 
against monomicrobial and polymicrobial biofilms $(0.5$ to 1 logs CFU reduction at $32 \mu \mathrm{g} / \mathrm{ml}$ ) compared to voriconazole alone but showed no statistical significance $(\mathrm{P}=0.5724)$.

Figure $4 \mathrm{~B}$ shows the effectiveness of voriconazole alone and in combination with cefepime against $A$. fumigatus monomicrobial and $A$. fumigatus- $P$. aeruginosa polymicrobial biofilms as determined by MTT assay. A comparison of the $\mathrm{A}_{570}$ values obtained for monomicrobial and polymicrobial biofilms as a function of voriconazole concentration showed that the polymicrobial biofilm is less susceptible to the fungicidal activity of the antifungal drug $(\mathrm{P}<0.01)$. Similarly, voriconazole in combination with cefepime was less active against polymicrobial biofilm compared to the activity against monomicrobial biofilm $(\mathrm{P}<0.01)$. This finding is contrary to what was obtained in the CFU assay where both monomicrobial and polymicrobial biofilms of $A$. fumigatus was almost equally susceptible to voriconazole with and without cefepime. Thus, the apparent resistance of A. fumigatus in polymicrobial biofilm to voriconazole may be an artifact of the MTT assay due to the presence of $P$. aeruginosa cells not susceptible to voriconazole but actively contributing to tetrazolium reduction in the polymicrobial biofilms. In support of this suggestion it was noted that a comparison of the effect of voriconazole alone and in combination with cefepime against monomicrobial biofilm is very similar $(P>0.05)$. Similarly, the effect of voriconazole alone and in combination with cefepime against $A$. fumigatus- $P$. aeruginosa biofilm is almost identical $(\mathrm{P}>0.05)$ showing no significant difference. Thus, since there is no suitable way of separating the fungal and the bacterial contributions to the tetrazolium reduction the MTT assay is unsuitable for studying the bioactivity of voriconazole against $A$. fumigatus biofilm.

Figure 5 shows the effects of cefepime and posaconazole individually and in combination on monomicrobial and polymicrobial biofilms of $P$. aeruginosa and $A$. fumigatus. A comparison of the susceptibilities of $A$. fumigatus monomicrobial and $A$. fumigatus-P. aeruginosa polymicrobial biofilms to posaconazole with and without cefepime (Panel A) provided 1 to 1.5 logs CFU reduction at a drug(s) concentration of $64 \mu \mathrm{g} / \mathrm{ml}$ and showed no significant difference $(\mathrm{P}>0.05)$. In contrast, a comparison of the effects of cefepime on $P$. aeruginosa monomicrobial $(\approx 4.5 \operatorname{logs}$ CFU reduction at a $64 \mu \mathrm{g} / \mathrm{ml})$ and $P$. aeruginosa- $A$. fumigatus polymicrobial $(\approx 1.5 \operatorname{logs} \mathrm{CFU}$ reduction at $64 \mu \mathrm{g} / \mathrm{ml}$ ) biofilms (Panel B) showed that the polymicrobial biofilm is significantly less susceptible to cefepime $(\mathrm{P}<0.0001)$. Similarly, a comparison of the effects of combination of cefepime with posaconazole on monomicrobial biofilm of $P$. aeruginosa $(\approx 4$ logs CFU reduction at $64 \mu \mathrm{g} / \mathrm{ml}$ ) with that obtained for polymicrobial biofilm $(\approx 1.5$ logs CFU reduction at $64 \mu \mathrm{g} / \mathrm{ml}$ ) showed that polymicrobial biofilm is also significantly less susceptible to the combination of drugs $(\mathrm{P}=0.0013)$. However, a comparison of the susceptibility of $P$. aeruginosa monomicrobial biofilm to cefepime alone $(\approx 4.5 \operatorname{logs}$ CFU reduction at a $64 \mu \mathrm{g} / \mathrm{ml})$ and cefepime plus posaconazole $(\approx 4 \operatorname{logs} \mathrm{CFU}$ reduction at $64 \mu \mathrm{g} / \mathrm{ml})$ showed no significant difference $(\mathrm{P}=0.4234)$ indicating that posaconazole has no detectable effect on the antibacterial activity of cefepime. Similarly, a comparison of the effect of cefepime on polymicrobial biofilm $(\approx 1.5 \operatorname{logs}$ CFU reduction at $64 \mu \mathrm{g} / \mathrm{ml})$ with that of the combination of cefepime and posaconazole $(\approx 1.5$ logs CFU reduction at $64 \mu \mathrm{g} / \mathrm{ml}$ ) showed that the polymicrobial biofilm was almost equally susceptible $(\mathrm{P}=$ 0.4057) to the drug combination suggesting that the presence of posaconazole in the combination did not affect bioactivity of cefepime against polymicrobial biofilm.

Since cefepime alone and in combination with posaconazole showed differential activity against $P$. aeruginosa monomicrobial and $P$. aeruginosa-A. fumigatus polymicrobial biofilms, we investigated the effect of tobramycin alone and in two-drug combination with posaconazole. As shown in Figure 6A, posaconazole with and without tobramycin was almost equally effective against both monomicrobial and polymicrobial biofilms with approximately 2 to 2.5 logs CFU reduction at a drug concentration of $64 \mu \mathrm{g} / \mathrm{ml}(\mathrm{P}>0.05)$. Similarly, Figure $6 \mathrm{~B}$ shows the effect of tobramycin alone and in combination with posaconazole against $P$. aeruginosa monomicrobial and $P$. aeruginosa- $A$. fumigatus polymicrobial biofilms. Tobramycin with and without posaconazole were equally active against the $P$. aeruginosa monomicrobial and $P$. aeruginosa- $A$. fumigatus polymicrobial biofilms with approximately 5-6 logs CFU reduction at a drug concentration of $64 \mu \mathrm{g} / \mathrm{ml}(\mathrm{P}>0.05)$. These results also show that tobramycin and posaconazole has no in vitro drugto-drug interaction to reduce the bioactivity of the other drug. The excellent activity of tobramycin against monomicrobial and polymicrobial biofilms is in sharp contrast to the differential effects of cefepime alone and in combination with posaconazole against monomicrobial and polymicrobial biofilms of $A$. fumigatus and $P$. aeruginosa.

\section{Discussion}

$P$. aeruginosa is known to produce an array of small molecules possessing antimicrobial activity by direct or indirect interaction with cells. So one of the intriguing questions is why $A$. fumigatus hyphae are refractory to the fungicidal effect of $P$. aeruginosa whereas conidia and sporelings are completely killed. Several reasons could be mentioned for the poor susceptibility of $A$. 


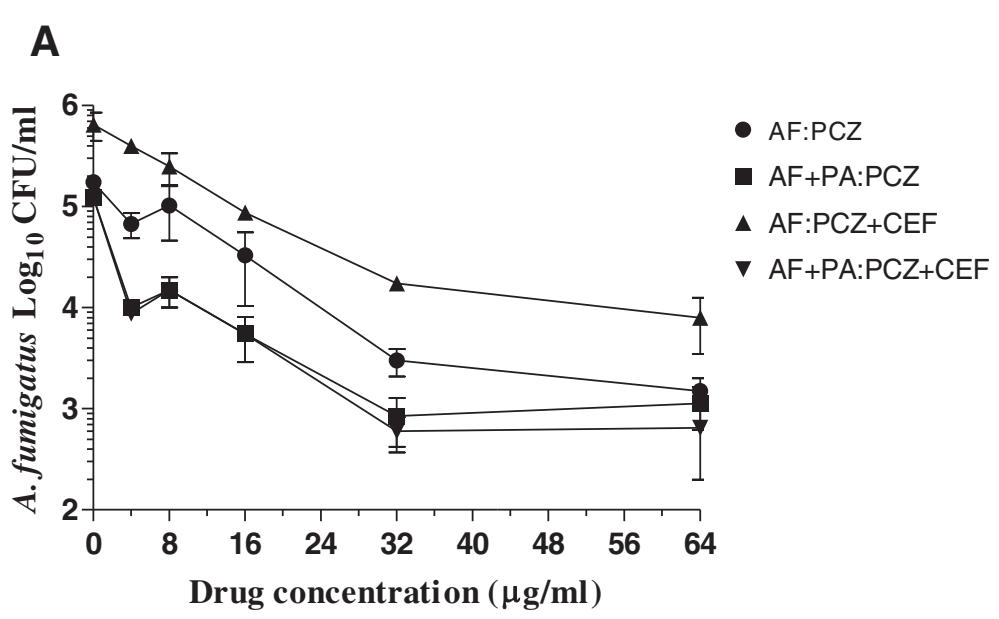

B

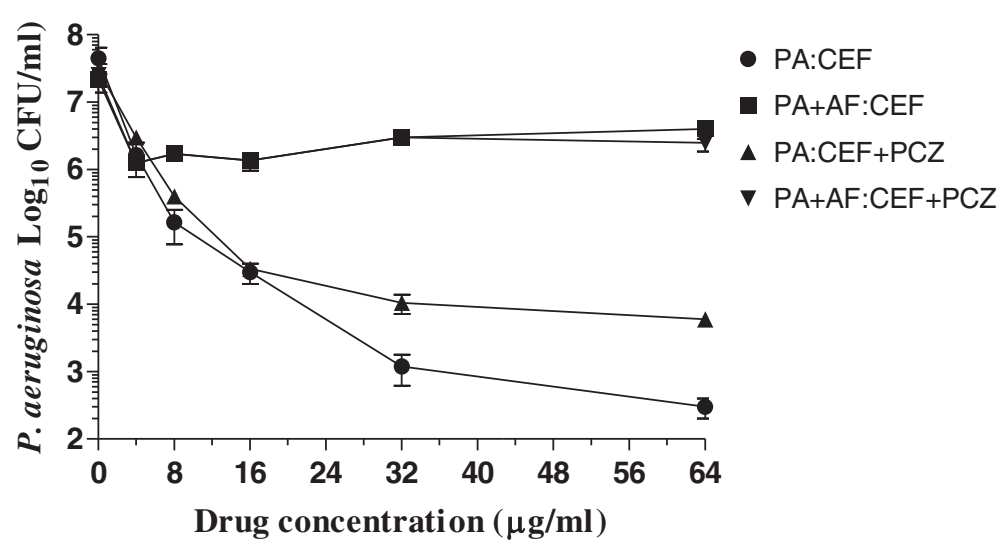

Figure 5 Biofilm inhibition by posaconazole and cefepime. A. Effects of posaconazole alone and in combination with cefepime against $A$. fumigatus monomicrobial and A. fumigatus-P. aeruginosa polymicrobial biofilms. B. Effects of cefepime alone and in combination with posaconazole against $P$. aeruginosa monomicrobial and $P$. aeruginosa-A. fumigatus polymicrobial biofilms. Each experiment was performed two different times with the clinical isolates AF53470 and PA57402 using independently prepared conidial suspensions and bacterial cultures, and one time with the laboratory isolates AF36607 and PA27853. Both clinical and laboratory isolates provided similar results. The data were analyzed by one-way and two-way ANOVA with Bonferroni's multiple comparison test where each set of data is compared with all the other sets of data as well as by paired two-tailed Student's t-test using Graphpad Prism 5.0. The vertical bar on each data point denotes standard error of the mean for two independent experiments performed with the clinical isolates. Legends: AF, A. fumigatus monomicrobial biofilm; PA, P. aeruginosa monomicrobial biofilm; PA + AF and $\mathbf{A F}+\mathbf{P A}$, polymicrobial biofilm; CEF, cefepime; PCZ, posaconazole.

fumigatus hyphae to the inhibitory effect of $P$. aeruginosa in mixed cultures: (1) Gliotoxin is a cytotoxic compound with antibacterial activity produced by $A$. fumigatus. The synthesis of this mycotoxin molecule is upregulated during mycelial growth in A. fumigatus, in particular during biofilm formation. So the increased level of gliotoxin during biofilm formation could inhibit $P$. aeruginosa growth or retards its ability to kill $A$. fumigatus. (2) It is generally known that metabolic activity of the cells is essential for $P$. aeruginosa virulence factors to be effective eliciting its inhibitory action. Germinating conidia and young sporelings are more or less uniformly metabolically active whereas in more mature hyphae metabolic activity is restricted to the apical regions of the filaments where hyphal extension takes place, although any part of growing hyphae is capable of regeneration (pluripotent) producing an actively growing fungal colony. Thus, the metabolically quiescent vegetative mycelia are less susceptible to the cytotoxic molecules produced by $P$. aeruginosa. (3) The cell wall chemistry of the mature hyphae is different from that of the young hyphae and the cell wall of matured hyphae may have restricted permeability to $P$. aeruginosa produced toxic molecules.

$P$. aeruginosa is a well known biofilm producer both in the laboratory and in clinical settings, especially in chronic infections [51-59]. One of the hallmarks of $P$. aeruginosa biofilm is its profound tolerance for 

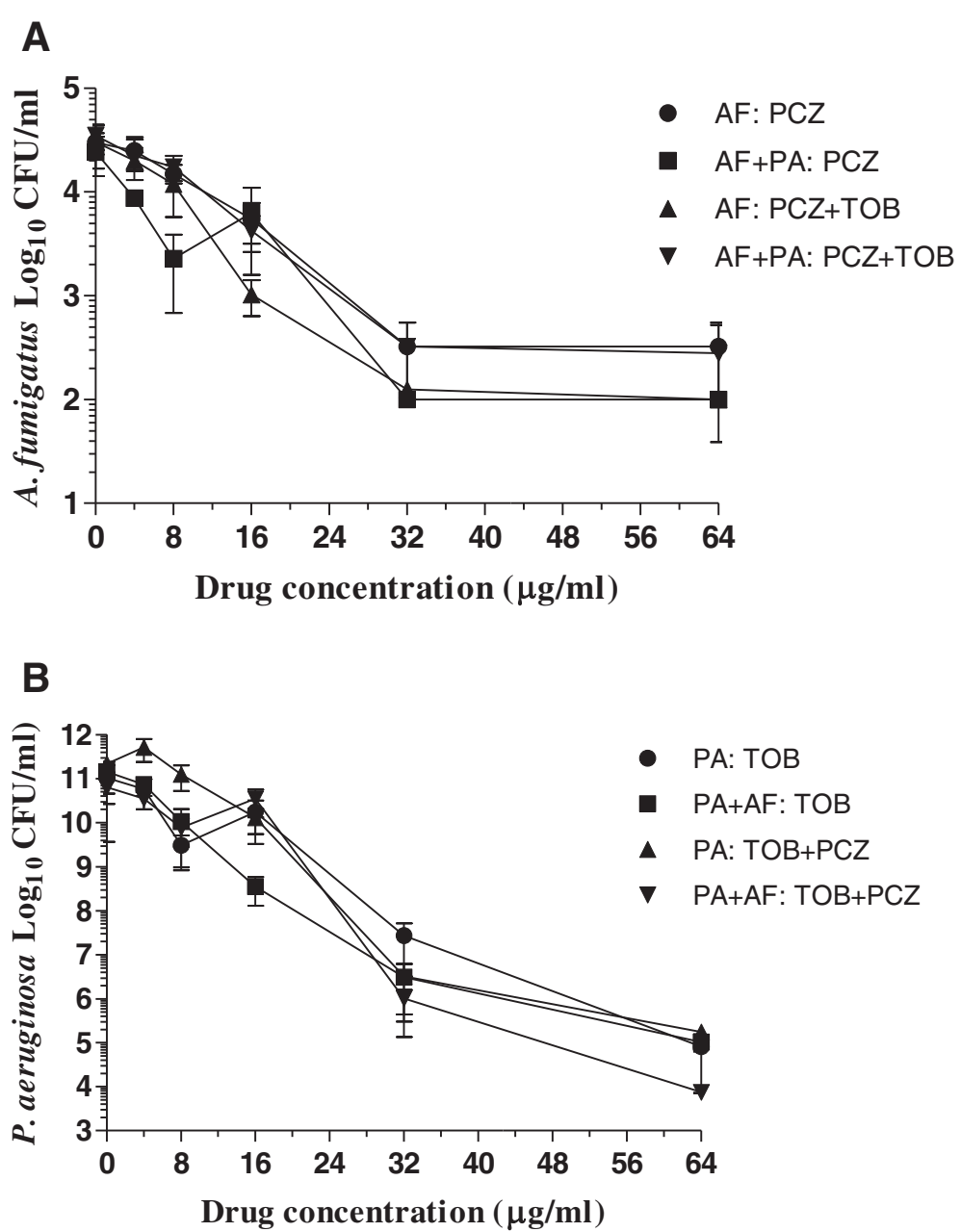

Figure 6 Biofilm inhibition by posaconazole and tobramycin. A. Effects of posaconazole alone and in combination with tobramycin against A. fumigatus monomicrobial and A. fumigatus-P. aeruginosa polymicrobial biofilms. B. Effects of tobramycin alone and in combination with posaconazole against $P$. aeruginosa monomicrobial and $P$. aeruginosa-A. fumigatus polymicrobial biofilms. Each experiment was performed two different times with the clinical isolates AF53470 and PA57402 using independently prepared conidial suspensions and bacterial cultures, and one time with the laboratory isolates AF36607 and PA27853. Both clinical and laboratory isolates provided similar results. The data were analyzed by one-way and two-way ANOVA with Bonferroni's multiple comparison test where each set of data is compared with all the other sets of data as well as by paired two-tailed Student's t-test using Graphpad Prism 5.0. The vertical bar on each data point denotes standard error of the mean for two independent experiments performed with the clinical isolates. Legends: AF, A. fumigatus monomicrobial biofilm; PA, P. aeruginosa monomicrobial biofilm; AF + PA and PA + AF, polymicrobial biofilm; PCZ, posaconazole; TOB, tobramycin.

antimicrobial drugs and microbiocidal agents while the individual cells of the biofilm community are highly drug susceptible in planktonic cultures [38,40,42,60,61]. Nearly four decades of research has provided a wealth of valuable information on the genesis, architecture, chemical composition and the drug susceptibility of $P$. aeruginosa biofilm [62,63]. In contrast, currently we know very little about $A$. fumigatus biofilm and the first report on A. fumigatus monomicrobial biofilm was published by Mowat et al. [40,60] in 2007. These investigators described that A. fumigatus forms an extensive net work of hyphae producing a multicellular community firmly attached to a solid substrate, and the adherent mycelial growth was encased in an extracellular matrix that resembles a biofilm microbial community. In addition, these investigators described that the extracellular matrix bound adherent fungal cells were highly resistant to antifungal drug treatment $[40,60,64]$ compared to their free-floating counter parts.

The high prevalence $[65,66]$ of $P$. aeruginosa and $A$. fumigatus in CF patients suffering from persistent lung infection provides a highly suitable ecological niche for the production of mixed microbial biofilm. The characteristics of polymicrobial biofilms produced by these organisms in mixed microbial cultures are largely unknown. Thus, the primary objective of our study was to develop a simple reliable easy to perform procedure for the development of a stably adhered polymicrobial 
biofilm of $A$. fumigatus and $P$. aeruginosa using mixed microbial culture of these organisms.

We examined several types of multi-well cell culture plates (6-well to 96-well) and growth media for the development of $A$. fumigatus- $P$. aeruginosa polymicrobial biofilm in cocultures. Although the 96-well cell culture plate would give a large number of replications for antimicrobial susceptibility studies, the wells in 96-well cell culture plates were found to be too small to prevent cross-contamination between wells by the surface growth of A. fumigatus. In contrast, the 6-well and 12well cell culture plates were found to be too big and comparatively large volumes of medium were needed for the development of biofilms and provided limited number of replications for drug susceptibility studies. In our experience, Costar 24-well cell culture plates were ideal for the development of in vitro monomicrobial and polymicrobial biofilms of $A$. fumigatus and $P$. aeruginosa and provided sufficient number of wells for replications. The large deep wells were adequately separated for multiple manipulations of the biofilm without crosscontamination between wells. In SD broth the 24-h and 48-h mixed microbial cultures of A. fumigatus and $P$. aeruginosa produced polymicrobial biofilms at $35^{\circ} \mathrm{C}$. Although the biofilm mass was significantly higher in $48 \mathrm{~h}$ biofilm, there was no significant difference for the CFU values obtained for the 24 -h and 48 -h cocultures. Therefore, we would suggest that $24 \mathrm{~h}$ growth of the mixed microbial culture will be sufficient to produce a functional $A$. fumigatus- $P$. aeruginosa polymicrobial biofilm for antimicrobial drug susceptibility studies.

The tetrazolium reduction assay has been used by several investigators in the past to examine the viability of a variety of eukaryotic cells ranging from mammalian to fungal cells, including members of the genus Aspergillus [48,67-71]. Therefore, we investigated the feasibility of using methyltetrazolium (MTT) assay for monitoring the viability of $A$. fumigatus cells after coculturing with $P$. aeruginosa in mixed microbial biofilms. The MTT assay has been used in our laboratory [68] previously, found to be convenient and highly sensitive for monitoring the viability of $A$. fumigatus cells, in particular after exposure to antifungal drugs. Similarly, we found in the current series of experiments that the MTT assay was very useful for monitoring the viability of A. fumigatus cells in monospecies cultures after $24 \mathrm{~h}$ and $48 \mathrm{~h}$ growth. However, in the mixed species cultures where $A$. fumigatus and $P$. aeruginosa were grown together in cocultures although the assay was highly sensitive and easy to perform, it was found to be difficult to distinguish the contribution made by the bacterial and fungal cells towards the reduction of the MTT compound. Therefore, we used only the CFU assay to monitor the growth of $A$. fumigatus cells in mixed microbial biofilms and for drug susceptibility studies. Apart from the inconvenience, the main disadvantages of using the CFU assay for determining the viability of $A$. fumigatus cells are the under estimation of CFUs due to clumping of hyphae and that the bacteria-treated fungal cells can be inhibited from growing without being killed. Since the end point of CFU assay is the formation of fungal colonies by individual cells, growth inhibition without killing would go undetected. Nonetheless, the fact that we washed the treated cells extensively with sterile distilled water makes it unlikely that in our experiments the fungal cells were only inhibited by the bacterial cells without killing them.

Our results show that the monomicrobial and the polymicrobial biofilms of $A$. fumigatus and A. fumigatus$P$. aeruginosa were almost equally susceptible to antifungal drugs such as voriconazole and posaconazole. The main reasons for the biofilm to exhibit drug resistance/ tolerance are (1) biofilm specific upregulation of efflux proteins (2) the presence of an extracellular matrix and (3) the presence of persistor cells that are inherently drug resistant/tolerant due to their low metabolic rate. It is likely that there is no differential upregulation of efflux proteins in monomicrobial and polymicrobial biofilms of A. fumigatus and A. fumigatus-P. aeruginosa. Similarly, although it is possible that the extracellular matrix produced by monomicrobial and polymicrobial biofilms of $A$. fumigatus and A. fumigatus-P. aeruginosa mixed culture is different, the difference in the permeability characteristics of monomicrobial and polymicrobial biofilm produced extracellular matrices are not sufficient enough to show any reduction in drug penetration. Since the growth characteristics and the biology of A. fumigatus is vastly different from other unicellular organisms such as bacteria and pathogenic yeasts, the presence of persistor cells inherently resistant to antimicrobial drug is highly unlikely. Together, these points suggest that although differential antifungal drug susceptibility for A. fumigatus monomicrobial and polymicrobial biofilms was expected, the lack of such response is not entirely surprising.

In contrast, our antimicrobial drug susceptibility studies showed that polymicrobial biofilm associated P. aeruginosa cells are less susceptible to cefepime in comparison to their monomicrobial counterparts. The extracellular matrix of $P$. aeruginosa biofilm is composed of proteins, polysaccharides, in particular alginate, and eDNA whereas that of A. fumigatus biofilm is made up of galactomannan, alpha-1,3 glucans, monosaccharides and polyols, pigments, proteins and eDNA. The most plausible explanation for the reduced susceptibility of polymicrobial biofilm embedded $P$. aeruginosa is the difference in the make up of the extracellular matrix of monomicrobial ( $P$. aeruginosa) and mixed microbial $(P$. aeruginosa- $A$. fumigatus) biofilms. The polymicrobial 
extracellular matrix may have permeability properties different from that of the monomicrobial extracellular matrix preventing adequate access to the biofilm embedded cells.

\section{Conclusions}

The high prevalence of $P$. aeruginosa and A. fumigatus colonization of the airways of CF patients results in mixed microbial chronic infections. The polymicrobial CF patient airway infection with $P$. aeruginosa and $A$. fumigatus produces mixed microbial biofilm with structural and functional characteristics different from those of monomicrobial biofilms. The monomicrobial extracellular matrix embedded bacterial and fungal cells are highly resistant to antimicrobial drug therapy. Although the formation of mixed microbial biofilm is considered to be a serious clinical problem in CF patients as well as in other patient groups prone to airway infection with $P$. aeruginosa and $A$. fumigatus, we know very little about the antibiotic susceptibility of $P$. aeruginosa-A. fumigatus polymicrobial biofilm. We therefore investigated the feasibility of developing an in vitro polymicrobial biofilm model using simultaneous static cocultures of A. fumigatus and $P$. aeruginosa for studying drug susceptibility. Simultaneous coculturing of $A$. fumigatus conidia with $P$. aeruginosa resulted in the complete killing of the fungus whereas A. fumigatus sporelings grown for $12 \mathrm{~h}$ or longer were recalcitrant to the fungicidal activity of $P$. aeruginosa and the young hyphae were highly suitable for producing sustainable polymicrobial biofilm with $P$. aeruginosa in cocultures. Using this in vitro model we studied the effects of cefepime and tobramycin alone and combination with posaconazole on monomicrobial and polymicrobial biofilms of $P$. aeruginosa and A. fumigatus. Our results show that $P$. aeruginosa cells associated with polymicrobial biofilm were less susceptible to cefepime (but not to tobramycin) compared to those of monomicrobial biofilm. On the other hand, A. fumigatus showed similar antifungal drug susceptibility in monomicrobial and polymicrobial biofilms.

\section{Abbreviations}

CF: Cystic fibrosis; SD: Sabouraud's dextrose; BHI: Brain Heart Infusion; RPMI: RPMI1640; CFU: Colony forming unit.

\footnotetext{
Authors' contributions

EKM together with JAV planned and designed all the experiments described in this manuscript, as well as performed the photomicrographic studies, experiments describing the biofilm assay, part of the drug susceptibility studies and the initial data analysis. Also, EKM prepared the initial draft of the manuscript. DLV performed all the experiments describing the interaction of germinated and ungerminated $A$. fumigatus conidia with $P$. aeruginosa cells, some of the drug susceptibility experiments as well as the effects of various microbial growth medium on the monomicrobial biofilm formation of $P$. aeruginosa cells on Costar tissue culture plates. JAV helped EKM in the planning and designing of all the experiments as well as performed analysis and interpretation of the results. Also, JAV revised the initial draft of the
}

manuscript and prepared the submitted version. All authors read and approved the final manuscript.

\section{Acknowledgements}

The authors would like to thank Dr. Dwayne Baxa, Division of Infectious Diseases, Henry Ford Hospital for assistance with photomicrography and SOPT Image Analysis Computer Program. This work was supported by Intramural Research Support from the Division of Infectious Diseases, Henry Ford Hospital, Detroit, Michigan, USA.

\section{Disclosures}

None of the authors has any conflict of interest for the work described in this manuscript.

Received: 21 November 2012 Accepted: 17 February 2014

Published: 3 March 2014

\section{References}

1. Zwielehner J, Lassl C, Hippe B, Pointner A, Switzeny OJ, Remely M, Kitzweger E, Ruckser R, Haslberger AG: Changes in human fecal microbiota due to chemotherapy analyzed by TaqMan-PCR, 454 sequencing and PCR-DGGE fingerprinting. PLOS One 2011, 6:e28654.

2. Charlson ES, Diamond JM, Bittinger K, Fitzgerald AS, Yadav A, Haas AR, Bushman FD, Collman RG: Lung-enriched organisms and aberrant bacterial and fungal respiratory microbiota after lung transplant. Am J Respir Crit Care Med 2012, 186:536-545.

3. Iwai S, Fei M, Huang D, Fong S, Subramanian A, Grieco K, Lynch SV, Huang L: Oral and airway microbiota in HIV-infected pneumonia patients. J Clin Microbiol 2012, 50:2995-3002.

4. Rolston KV, Bodey GP, Safdar A: Polymicrobial infection in patients with cancer: an underappreciated and underreported entity. Clin Infect Dis 2007, 45:228-233.

5. Duggal R, Rajwanshi A, Gupta N, Lal A, Singhal M: Polymicrobial lung infection in postrenal transplant recipient diagnosed by fine-needle aspiration cytology. Diagn Cytopathol 2010, 38:294-296.

6. Tuttle MS, Mostow E, Mukherjee P, Hu FZ, Melton-Kreft R, Ehrlich GD, Dowd SE, Ghannoum MA: Characterization of bacterial communities in venous insufficiency wounds by use of conventional culture and molecular diagnostic methods. J Clin Microbiol 2011, 49:3812-3819.

7. Grice EA, Snitkin ES, Yockey L, Bermudez DM, Liechty KW, Segre JA: Longitudinal shift in diabetic wound microbiota correlates with prolonged skin defense response. Proc Natl Acad Sci U S A 2010, 107:14799-14804.

8. Scales BS, Huffnagle GB: The microbiome in wound repair and tissue fibrosis. J Pathol 2013, 229:323-331. doi:10.1002/path.4118.

9. Kirkup BC Jr, Craft DW, Palys T, Black C, Heitkamp R, Li C, Lu Y, Matlock N, McQueary C, Michels A, Peck G, Si Y, Summers AM, Thompson M, Zurawski DV: Traumatic wound microbiome workshop. Microb Ecol 2012, 64:837-850

10. Erb-Downward JR, Thompson DL, Han MK, Freeman CM, McCloskey L, Schmidt LA, Young VB, Toews GB, Curtis JL, Sundaram B, Martinez FJ, Huffnagle GB: Analysis of the lung microbiome in the "healthy" smoker and in COPD. PLoS One 2011, 6:e16384.

11. Pragman AA, Kim HB, Reilly CS, Wendt C, Isaacson RE: The lung microbiome in moderate and severe chronic obstructive pulmonary disease. PLoS One 2012, 7:e47305

12. Sze MA, Dimitriu PA, Hayashi S, Elliott WM, McDonough JE, Gosselink JV, Cooper J, Sin DD, Mohn WW, Hogg JC: The lung tissue microbiome in chronic obstructive pulmonary disease. Am J Respir Crit Care Med 2012, 185:1073-1080.

13. Cabrera-Rubio R, Garcia-Nunez M, Seto L, Anto JM, Moya A, Monso E, Mira $A$ : Microbiome diversity in the bronchial tracts of patients with chronic obstructive pulmonary disease. J Clin Microbiol 2012, 50:3562-3568.

14. Zemanick ET, Sagel SD, Harris JK: The airway microbiome in cystic fibrosis and implications for treatment. Curr Opin Pediatr 2011, 23:319-324.

15. Stressmann FA, Rogers GB, Klem ER, Lilley AK, Donaldson SH, Daniels TW, Carroll MP, Patel N, Forbes B, Boucher RC, Wolfgang MC, Bruce KD: Analysis of the bacterial communities present in lungs of patients with cystic fibrosis from American and British centers. J Clin Microbiol 2011, 49:281-291 
16. Rogers GB, Carroll MP, Hoffman LR, Walker AW, Fine DA, Bruce KD: Comparing the microbiota of the cystic fibrosis lung and human gut. Gut Microbes 2010, 1:85-93.

17. Guss AM, Roeselers G, Newton IL, Young CR, Klepac-Ceraj V, Lory S, Cavanaugh CM: Phylogenetic and metabolic diversity of bacteria associated with cystic fibrosis. ISME J 2011, 5:20-29.

18. Sibley CD, Surette MG: The polymicrobial nature of airway infections in cystic fibrosis: cangene gold medal lecture. Can J Microbiol 2011, 57:69-77.

19. Madan JC, Koestler DC, Stanton BA, Davidson L, Moulton LA, Housman ML, Moore JH, Guill MF, Morrison HG, Sogin ML, Hampton TH, Karagas MR, Palumbo PE, Foster JA, Hibberd PL, OToole GA: Serial analysis of the gut and respiratory microbiome in cystic fibrosis in infancy: interaction between intestinal and respiratory tracts and impact of nutritional exposures. MBio 2012, 3(4):e00251-12. doi:10.1128/mBio.00251-12.

20. Amin R, Dupuis A, Aaron SD, Ratjen F: The Effect of chronic infection with Aspergillus fumigatus on lung function and hospitalization in patients with cystic fibrosis. Chest 2011, 137:171-176.

21. Kanj SS, Tapson V, Davis RD, Madden J, Browning I: Infections in patients with cystic fibrosis following lung transplantation. Chest 1997 112:924-930.

22. Helmi M, Love RB, Welter D, Cornwell RD, Meyer KC: Aspergillus infection in lung transplant recipients with cystic fibrosis: risk factors and outcomes comparison to other types of transplant recipients. Chest 2003, 123:800-808.

23. Iversen M, Burton CM, Vand S, Skovfoged L, Carlsen J, Milman N, Andersen $C B$, Rasmussen $M$, Tvede M: Aspergillus infection in lung transplant patients: incidence and prognosis. Eur J Clin Microbiol Infect Dis 2007, 26:879-886

24. Razvi S, Quittell L, Sewall A, Quinton H, Marshall B, Saiman L: Respiratory microbiology of patients with cystic fibrosis in the United States, 1995 to 2005. Chest 2009, 136:1554-1560

25. Lipuma JJ: The changing microbial epidemiology in cystic fibrosis. Clin Microbiol Rev 2010, 23:299-323.

26. Pihet M, Carrere J, Cimon B, Chabasse D, Delhaes L, Symoens F, Bouchara JP: Occurrence and relevance of filamentous fungi in respiratory secretions of patients with cystic fibrosis-a review. Med Mycol 2009, 47:387-397.

27. Hauser AR, Jain M, Bar-Meir M, McColley SA: Clinical significance of microbial infection and adaptation in cystic fibrosis. Clin Microbiol Rev 2011, 24:29-70.

28. Foundation CF: Patient Registry 2008: Annual Data Report to the Center Directors. Bethesda, MD: Cystic Fibrosis Foundation; 2008.

29. Valenza G, Tappe D, Turnwald D, Frosch M, Konig C, Hebestreit H, AbeleHorn M: Prevalence and antimicrobial susceptibility of microorganisms isolated from sputa of patients with cystic fibrosis. J Cyst Fibros 2008, 7:123-127.

30. Bakare N, Rickerts V, Bargon J, Just-Nubling G: Prevalence of Aspergillus fumigatus and other fungal species in the sputum of adult patients with cystic fibrosis. Mycoses 2003, 46:19-23.

31. Patterson K, Strek ME: Allergic bronchopulmonary aspergillosis. Proc Am Thorac Soc 2010, 7:237-244.

32. Moss RB: Allergic bronchopulmonary aspergillosis and Aspergillus infection in cystic fibrosis. Curr Opin Pulm Med 2010, 16:598-603.

33. Kraemer R, Delosea N, Ballinari P, Gallati S, Crameri R: Effect of allergic bronchopulmonary aspergillosis on lung function in children with cystic fibrosis. Am J Respir Crit Care Med 2006, 174:1211-1220.

34. Jubin V, Ranque S, Stremler Le Bel N, Sarles J, Dubus JC: Risk factors for Aspergillus colonization and allergic bronchopulmonary aspergillosis in children with cystic fibrosis. Pediatr Pulmonol 2010, 45:764-771.

35. Moore JE, Shaw A, Millar BC, Downey DG, Murphy PG, Elborn JS: Microbial ecology of the cystic fibrosis lung: does microflora type influence microbial loading? Br J Biomed Sci 2005, 62:175-178.

36. Millar FA, Simmonds NJ, Hodson ME: Trends in pathogens colonising the respiratory tract of adult patients with cystic fibrosis, 1985-2005. J Cyst Fibros 2009, 8:386-391.

37. Hoiby N, Bjarnsholt T, Givskov M, Molin S, Ciofu O: Antibiotic resistance of bacterial biofilms. Int J Antimicrob Agents 2010, 35:322-332.

38. Seidler MJ, Salvenmoser S, Muller FM: Aspergillus fumigatus forms biofilms with reduced antifungal drug susceptibility on bronchial epithelial cells. Antimicrob Agents Chemother 2008, 52:4130-4136.
39. Olson ME, Ceri H, Morck DW, Buret AG, Read RR: Biofilm bacteria: formation and comparative susceptibility to antibiotics. Can J Vet Res 2002, 66:86-92.

40. Mowat E, Butcher J, Lang S, Williams C, Ramage G: Development of a simple model for studying the effects of antifungal agents on multicellular communities of Aspergillus fumigatus. J Med Microbiol 2007, 56:1205-1212.

41. Beauvais A, Schmidt C, Guadagnini S, Roux P, Perret E, Henry C, Paris S, Mallet A, Prevost MC, Latge JP: An extracellular matrix glues together the aerial-grown hyphae of Aspergillus fumigatus. Cell Microbiol 2007 9:1588-1600.

42. Loussert C, Schmitt C, Prevost MC, Balloy V, Fadel E, Philippe B, KauffmannLacroix C, Latge JP, Beauvais A: In vivo biofilm composition of Aspergillus fumigatus. Cell Microbiol 2010, 12:405-410.

43. Bruns S, Seidler M, Albrecht D, Salvenmoser S, Remme N, Hertweck C, Brakhage AA, Kniemeyer O, Muller FM: Functional genomic profiling of Aspergillus fumigatus biofilm reveals enhanced production of the mycotoxin gliotoxin. Proteomics 2010, 10:3097-3107.

44. Mowat E, Rajendran R, Williams C, McCulloch E, Jones B, Lang S, Ramage G Pseudomonas aeruginosa and their small diffusible extracellular molecules inhibit Aspergillus fumigatus biofilm formation. FEMS Microbiol Lett 2010, 313:96-102.

45. Moree WJ, Phelen W, Wu CH, Bandeira N, Cornett DS, Duggan BM, Dorrestein PC: Interkingdom metabolic transformations captured by microbial imaging mass spectrometry. Proc Natl Acad Sci U S A 2012, 109:13811-13816.

46. Software SC: SPOT Basic Software User Guide (Version 4.7) 2008, SPOT Imaging Solutions, A Division of Diagnostic Instruments, Inc. Sterling Heights, Ml 48314, USA.

47. Jahn B, Martin E, Stueben A, Bhakdi S: Susceptibility testing of Candida albicans and Aspergillus species by a simple microtiter menadioneaugmented 3-(4,5-dimethyl-2-thiazolyl)-2,5-diphenyl-2H-tetrazolium bromide assay. J Clin Microbiol 1995, 33:661-667.

48. Meletiadis J, Meis JF, Mouton JW, Donnelly JP, Verweij PE: Comparison of NCCLS and 3-(4,5-dimethyl-2-Thiazyl)-2, 5-diphenyl-2H-tetrazolium bromide (MTT) methods of in vitro susceptibility testing of filamentous fungi and development of a new simplified method. J Clin Microbiol 2000, 38:2949-2954

49. Horne MK: The adsorption of thrombin to polypropylene tubes: the effect of polyethylene glycol and bovine serum albumin. Thromb Res 1985, 37:201-212.

50. Hammond A, Dertien J, Colmer-Hamood JA, Griswold JA, Hamood AN: Serum inhibits $P$. aeruginosa biofilm formation on plastic surfaces and intravenous catheters. J Surg Res 2010, 159:735-746.

51. Gillis RJ, Iglewski BH: Azithromycin retards Pseudomonas aeruginosa biofilm formation. J Clin Microbiol 2004, 42:5842-5845.

52. Dales L, Ferris W, Vandemheen K, Aaron SD: Combination antibiotic susceptibility of biofilm-grown Burkholderia cepaca and P. aeruginosa isolated from patients with pulmonary exacerbations of cystic fibrosis. Eur J Clin Microbiol Infect Dis 2009, 28:1275-1279.

53. Mulcahy LR, Burns $J$, Lory S, Lewis K: Emergence of Pseudomonas aeruginosa strains producing high levels of persister cells in patients with cystic fibrosis. J Bacteriol 2010, 192:6191-6199.

54. Sadovskaya I, Vinogradov E, Li J, Hachani A, Kowalska K, Filloux A: High-level antibiotic resistance in Pseudomonas aeruginosa biofilm: the ndvB gene is involved in the production of highly glycerol-phosphorylated beta-(1>3)-glucans, which bind aminoglycosides. Glycobiology 2010, 20:895-904.

55. Tre-Hardy M, Vanderbist F, Traore H, Devleeschouwer MJ: In vitro activity of antibiotic combinations against Pseudomonas aeruginosa biofilm and planktonic cultures. Int J Antimicrob Agents 2008, 31:329-336.

56. Zhang L, Mah TF: Involvement of a novel efflux system in biofilm-specific resistance to antibiotics. J Bacteriol 2008, 190:4447-4452.

57. Mah TF, O'Toole GA: Mechanisms of biofilm resistance to antimicrobial agents. Trends Microbiol 2001, 9:34-39.

58. Wagner VE, Iglewski BH: P. aeruginosa biofilms in CF infection. Clin Rev Allergy Immunol 2008, 35:124-134

59. Drenkard E, Ausubel FM: Pseudomonas biofilm formation and antibiotic resistance are linked to phenotypic variation. Nature 2002, 416:740-743.

60. Mowat E, Williams C, Jones B, McChlery S, Ramage G: The characteristics of Aspergillus fumigatus mycetoma development: is this a biofilm? Med Mycol 2009, 47(Suppl 1):S120-S126. 
61. Ramage G, Mowat E, Jones B, Williams C, Lopez-Ribot J: Our current understanding of fungal biofilms. Crit Rev Microbiol 2009, 35:340-355.

62. Toutain CM, Caiazza N. C., O,Toole, G. A: Molecular Basis of Biofilm Development by Pseudomonads. In Microbial Biofilms. Edited by Ghannoum M, G A O,Toole. Washington, DC, USA: ASM Press, American Society for Microbiology; 2004:43-63.

63. Costerton JW: A Short History of the Development of the Biofilm Concept. In Microbial Biofilms. Edited by Ghannoum M, Toole GA. Washington, DC, USA: ASM Press, American Society for Microbiology; 2004:4-19.

64. Mowat E, Lang S, Williams C, McCulloch E, Jones B, Ramage G: Phasedependent antifungal activity against Aspergillus fumigatus developing multicellular filamentous biofilms. J Antimicrob Chemother 2008, 62:1281-1284.

65. Campos S, Caramori M, Teixeira R, Afonso J Jr, Carraro R, Strabelli T, Samano $M$, Pego-Fernandes $P$, Jatene F: Bacterial and fungal pneumonias after lung transplantation. Transplant Proc 2008, 40:822-824.

66. Leclair LW, Hogan DA: Mixed bacterial-fungal infections in the CF respiratory tract. Med Mycol 2010, 48(Suppl 1):S125-S132.

67. Petraitis V, Petraitiene R, Sarafandi AA, Kelaher AM, Lyman CA, Casler HE, Sein T, Groll AH, Bacher J, Avila NA, Walsh TJ: Combination therapy in treatment of experimental pulmonary aspergillosis: synergistic interaction between an antifungal triazole and an echinocandin. J Infect Dis 2003, 187:1834-1843.

68. Manavathu EK, Alangaden GJ, Chandrasekar PH: Differential activity of triazoles in two-drug combinations with the echinocandin caspofungin against Aspergillus fumigatus. J Antimicrob Chemother 2003, 51:1423-1425

69. Chen L, Shen Z, Wu J: Expression, purification and in vitro antifungal activity of acidic mammalian chitinase against Candida albicans, Aspergillus fumigatus and Trichophyton rubrum strains. Clin Exp Dermatol 2009, 34:55-60.

70. Lupetti A, van Dissel JT, Brouwer CP, Nibbering PH: Human antimicrobial peptides' antifungal activity against Aspergillus fumigatus. Eur J Clin Microbiol Infect Dis 2008, 27:1125-1129.

71. Chiou CC, Mavrogiorgos N, Tillem E, Hector R, Walsh TJ: Synergy, pharmacodynamics, and time-sequenced ultrastructural changes of the interaction between nikkomycin Z and the echinocandin FK463 against Aspergillus fumigatus. Antimicrob Agents Chemother 2001, 45:3310-3321.

doi:10.1186/1471-2180-14-53

Cite this article as: Manavathu et al:: Development and antimicrobial susceptibility studies of in vitro monomicrobial and polymicrobial biofilm models with Aspergillus fumigatus and Pseudomonas aeruginosa. BMC Microbiology 2014 14:53.

\section{Submit your next manuscript to BioMed Central and take full advantage of:}

- Convenient online submission

- Thorough peer review

- No space constraints or color figure charges

- Immediate publication on acceptance

- Inclusion in PubMed, CAS, Scopus and Google Scholar

- Research which is freely available for redistribution

Submit your manuscript at www.biomedcentral.com/submit
C Biomed Central 